Original article

\title{
Prevalence and predictors of musculoskeletal disorders (MSDs) among weavers of Varanasi, India: A cross-sectional study
}

\author{
Laeek Ahemad Siddiqui $^{\text {a, }}{ }^{*}$, Adrita Banerjee ${ }^{\mathrm{a}}$, Praveen Chokhandre ${ }^{\mathrm{b}}$, Sayeed Unisa ${ }^{\mathrm{a}}$

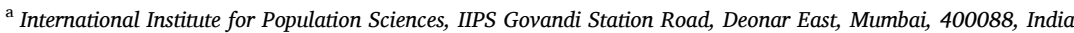 \\ ${ }^{\mathrm{b}}$ Population Research Centre (PRC), Dharwad, Karnataka, 580004, India
}

\section{A R T I C L E I N F O}

\section{Keywords:}

Musculoskeletal disorder (MSDs)

Textile industry

Handloom weavers

Powerloom weavers

India

\begin{abstract}
A B S T R A C T
Background: Work-related musculoskeletal disorders are widespread among workers in the weaving industry. The most affected body regions are the neck, shoulders, back, and wrist. The present study attempts to understand risk factors for MSDs in various body region among handloom and power loom weavers.

Method: A cross-sectional household survey was conducted on a sample of 364 weavers during November 2019 to February 2020. The survey instrument for measuring musculoskeletal symptoms was adopted from a standardised Nordic questionnaire. The impact of the occupation of weaving on MSDs was analysed using binary logistics regression.

Results: The 12-month prevalence of MSDs was higher among handloom weavers compared to power loom weavers, particularly in the upper back (84-45\%), lower back (82-50\%), knee (60-35\%), and shoulder (76-42\%) respectively. Similar patterns were observed in the 12-month prevalence of MSDs, which prevented regular activity inside or outside the home. Analysis suggests that the occupation of weaving raises the risk of MSDs particularly in the shoulder (OR: 4.52, C.I.: 2.63-7.76), lower (OR: 6.9, C.I.: 3.79-12.54) and upper back (OR: 5.63, C.I.: 3.19-9.95). Older age and long years of working are significant risk factors for MSDs.

Conclusions: The findings suggest a relatively higher prevalence of MSDs among handloom weavers, particularly in the upper back, lower back and shoulder, compared to Powerloom weavers. Preventive measures and treatment to minimise the burden of MSDs among handloom weavers are strongly recommended.
\end{abstract}

\section{Introduction}

India has always been known for its splendid crafts and Indian handlooms have attracted attention among the people all over the world. This sector is an informal sector where workers have long working hours, extremely low wages, without any job security and social security benefits, with unsatisfactory working and living conditions. Handloom weaving involves numerous activities which includes raw material (yarn) collection, winding, denting and then continuously sitting in static posture to weave fabric. ${ }^{1}$ The process of weaving encompasses a multitude of activities involving repeated movement of upper and lower limbs to operate pedals and shuttles, with arms rose away from the body. ${ }^{2}$ As a result of this weaving and other handloom activities become a high risk occupation for developing musculoskeletal disorders. ${ }^{3}$ Work-related musculoskeletal disorders (WMSDs) are the painful disorders often caused by overuse of the muscles, joints, nerves, tendons and soft tissues of the body. WMSDs are accounted for one of the costly occupational disorders because of its consequential impact on worker's health and productivity at work. ${ }^{4}$ MSDs are a major cause of morbidity and in many countries have emerged as the leading cause of occupational injury, illness and disability. ${ }^{5,6}$ Globally the burden of MSDs led WHO to declare 2000-2010 as the Bone and Joint Decade. ${ }^{7}$ Earlier literature based on handloom workers of Bangladesh have concluded that work related musculoskeletal disorders were widespread among the workers and the most affected body regions were neck, shoulder, back and wrist. ${ }^{8}$ Past studies based on the handloom workers have shown widespread prevalence of MSD among the workers mostly attributable to the working condition and aggravated by work related stress. $^{9-11}$ Different studies on workers of weaving industry of India reported that workers were having significant MSDs related to work. $^{2,3,12,13}$

The economic loss due to such diseases not only affects the

\footnotetext{
* Corresponding author.

E-mail addresses: siddiqui.laeek@gmail.com (L.A. Siddiqui), adrita.banerjee@yahoo.co.in (A. Banerjee), praveenchokhandre@gmail.com (P. Chokhandre), sayeed.unisa@iipsindia.ac.in (S. Unisa).
} 
individual, but also the organizational level and company as a whole. Studies on MSDs in the handloom as well as power loom industry are very rare in Indian context. In light of this situation, the present study focuses on identifying the WMSDs among the handloom and power loom workers. The primary objective of the study is to investigate the prevalence of MSDs among both the handloom and powerloom workers of Varanasi district of Uttar Pradesh. Additionally, an attempt has been made to identify potential risk factors that raised the risk of MSDs among these craftsmen. The main hypothesis of the study is that those engaged in handloom weaving are at greater risk of developing MSDs than power loom workers.

\section{Material and methods}

\subsection{Data source}

The data published by All India Handloom Census 2019-2020 shows that Uttar Pradesh had about 1,90,957 handloom workers. ${ }^{14}$ However the census does not provide data on the occupational health of the weavers. Hence this study was carried out in one of the oldest weaving hub in India, Varanasi, a district of Uttar Pradesh to assess the prevalence of MSDs among handloom and powerloom weaver. The exposed population consisted of weavers engaged in handloom industry for at least 10 years. Weavers engaged in power loom industry for at least 10 years served as the comparison group and lived in or close to the handloom weavers and in similar socio-economic conditions. Most of the respondents were daily wage labourers working in Varanasi Saree weaving industry and their workplace i.e. the handloom and power loom workshops were mostly in close vicinity of their home.

\subsection{Sampling procedure}

The estimated sample size for the present study was 364 with a prevalence rate of 0.17 and a design effect of 1.5. Data has been collected through multi-stage random sampling. Varanasi district consist of eight blocks. Since there is no readily available information on weaver's population size in these blocks, therefore present study has considered the Indian Census 2011. The Census provides occupational information as main and marginal workers only. These main workers consist of agricultural labourers, cultivators, household industry workers, other workers and non-workers. Since our study is based on weavers hence household industry worker and other worker has been considered for the present study, and rest were excluded to estimate the tentative number of weavers. Insights from preliminary field visits suggest that there were hardly any weaver community staying in the two blocks namely Sevapuri and Baragaon and hence excluded. Finally, two out of six blocks were randomly selected using probability proportional to size (PPS) sampling (namely Arajiline and Kashividyapeeth).

At the second level, villages and Census Towns were selected. Five villages were randomly selected from both the blocks using PPS sampling method. In the similar manner, two census town have been selected from each block. In the third stage, mapping and house listing operation was carried out in the selected villages to get a sampling frame for households having at least one person aged $\geq 50$ years, engaged or ever worked in weaving industry. In Census towns, several densely populated pockets were identified after interacting with key informants. Finally, estimated sample size was drawn according to the proportion of eligible weavers found at each village/Census Town using systematic random sampling (SRS). From every selected household one eligible respondent was interviewed for data collection based on face-to-face

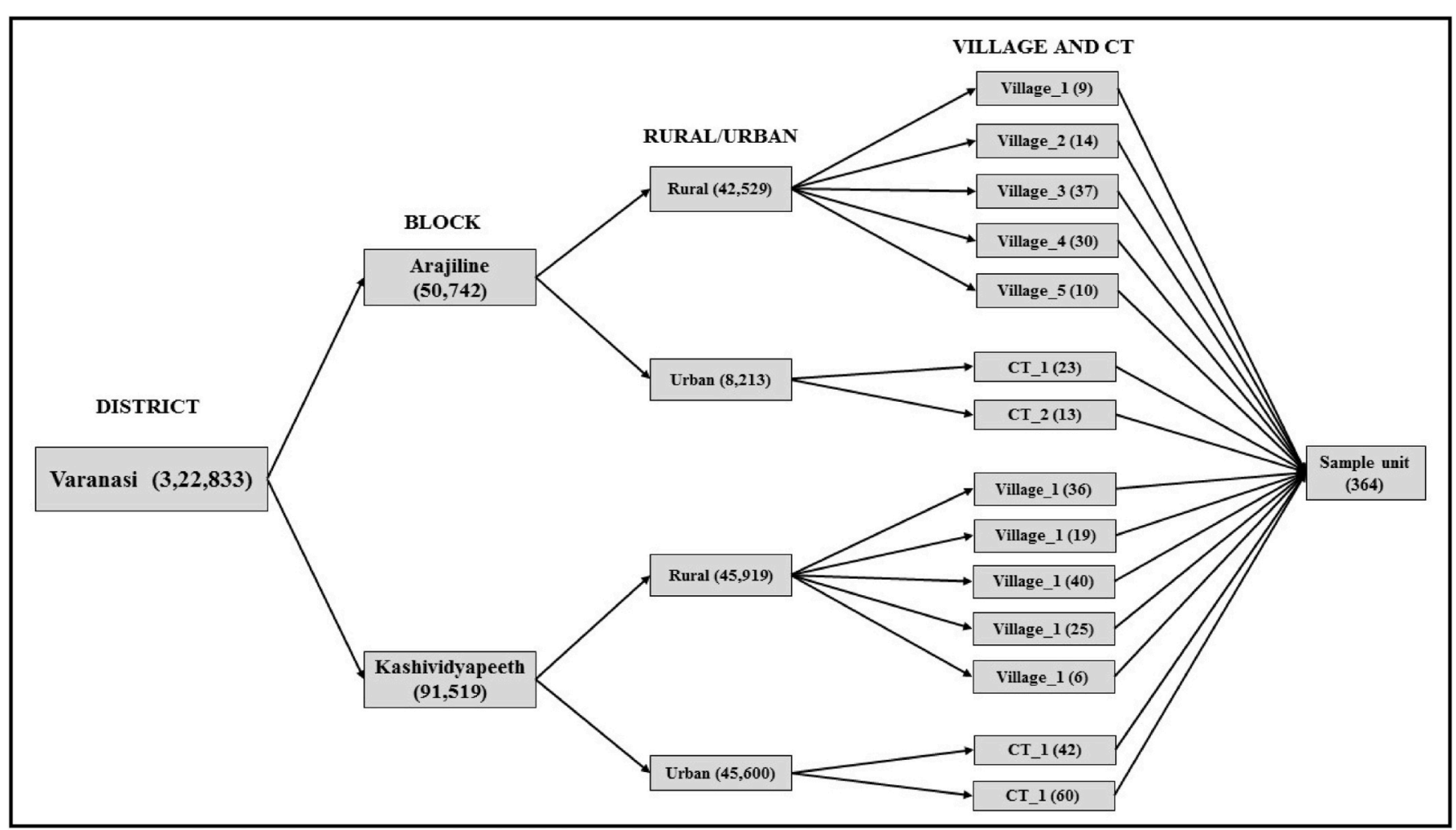

Note All the numbers indicate the Main worker population (Household Industry workers \& other workers) from Census 2011

Village and CT=Villages and Census Towns

In village and CT panel=Numbers are suggesting sample of weavers drawn from respective villages

Sample unit $=$ Sample size

Fig. 1. Sampling Framework.

Note All the numbers indicate the Main worker population (Household Industry workers \& other workers) from Census 2011. Village and CT=Villages and Census Towns. In village and CT panel = Numbers are suggesting sample of weavers drawn from respective villages. Sample unit = Sample size. 
interviews, using structured interview schedule. Finally, a total of 364 weavers were interviewed (226 from rural and 138 from urban setting) during November 2019 to February 2020 (see Fig. 1).

\subsection{Study tools and methods}

Our survey instrument for measuring musculoskeletal symptoms was adapted from the Standardized Nordic Questionnaire. ${ }^{15}$ and translated into Hindi language. An anatomical diagram with labels and arrows clearly indicating different body parts was used for assessing musculoskeletal symptoms. Information on musculoskeletal symptoms, and occupational and demographic characteristics was collected from respondents. Descriptive statistics were used to summarise the results. The prevalence of musculoskeletal symptoms that prevented normal work inside or outside the home was calculated for the handloom weavers and power loom weavers. Differences in the prevalence of MSDs among the groups were tested using the $\chi 2$ test.

\subsection{Variables}

\subsubsection{Outcome variable}

Respondents who have reported pain in the neck, hands, upper and lower back, thigh, knees or ankle in the past 12 months were considered to have MSD. In addition, inability to do normal work (inside or outside the home) in the past 12 months due to MSD were considered as response variable.

\subsubsection{Risk factor}

Handloom is a machine run manually with hand and foot combination. The workers perform strenuous activities such as manual handling and long hours of bending forward, which may compress tendons and nerves and lead to complaints of MSDs. Previous studies have indicated that activities which involve heavy use of hands, shoulder and legs with long working hours of repetitive task are at higher risk of MSDs. ${ }^{1,2,16-18}$ Align to the objective of the study, the paper tries to see at what extent, weaving occupation poses risk to workers by the method of weaving.

\subsubsection{Confounding factors}

The selected confounding factors include age-group (50-54 years and 55-65 years), work profile (worker; own workshop, other activities related to weaving), work satisfaction (yes, no) and years of working (11-24; 25-34; 35-45 years). Earlier studies have shown that complaints of MSDs were exclusively identified to be related with age. ${ }^{2,16,19}$ Similarly, studies have also suggested that long duration of work increases the MSD among the workers engaged in physical activity. ${ }^{20,21}$ Earlier studies have also indicated that job dissatisfaction, lack of support from employer had positive impact on MSDs. ${ }^{2,21}$

For identifying the covariates affecting MSDs and related disabilities among handloom as well as power loom workers, the multiple logistic regression analysis was employed. For a given occupation, the effect of risk factors (duration of occupation and age) on the incidence of MSDs among workers was established by applying multivariate logistic regression. Here, type of occupation was considered the exposure variable, the confounding factors were duration of work and age, and socioeconomic and demographic characteristics were controlled. The standard of living index has been classified into low, medium and high on the basis of available assets using principal component analysis technique. All the analysis was performed using STATAV.16.1 software.

\section{Results}

Table 1 shows the socio-economic and occupational profile of the handloom and power loom workers. Majority (44.02\%) of the handloom workers were in the age group of 50-54 years compared to power loom workers. The mean age of the handloom workers were 54.65 years and those of the power loom workers were 55.09 years. Most of the
Table 1

Socio-demographic and occupational profiles of the study groups.

\begin{tabular}{|c|c|c|}
\hline Background Variable & $\begin{array}{l}\text { Handloom Worker }(\mathrm{N}= \\
234)\end{array}$ & $\begin{array}{l}\text { Powerloom Worker }(\mathrm{N}= \\
\text { 102) }\end{array}$ \\
\hline \multicolumn{3}{|l|}{ Respondent age } \\
\hline $50-54$ age & 44.02 & 40.2 \\
\hline $56-65$ age & 55.98 & 59.8 \\
\hline Mean age $\pm S D$ & $54.65 \pm 3.15$ & $55.09 \pm 3.27$ \\
\hline \multicolumn{3}{|l|}{ Education } \\
\hline No education & 44.44 & 37.25 \\
\hline Up to primary & 48.72 & 55.88 \\
\hline Secondary \& above & 6.84 & 6.86 \\
\hline \multicolumn{3}{|l|}{ Family size } \\
\hline Less than 7 member & 23.5 & 30.39 \\
\hline 8 to 12 member & 48.29 & 38.24 \\
\hline 13 and above member & 28.21 & 31.37 \\
\hline Mean size of family \pm SD & $10.49 \pm 3.00$ & $10.52 \pm 3.18$ \\
\hline \multicolumn{3}{|l|}{ Religion } \\
\hline Hindu & 8.12 & 13.73 \\
\hline Muslim & 91.88 & 86.27 \\
\hline \multicolumn{3}{|l|}{ Type of family } \\
\hline Nuclear family & 74.36 & 80.39 \\
\hline Joint family & 25.64 & 19.61 \\
\hline \multicolumn{3}{|l|}{ Work profile } \\
\hline Worker & 24.89 & 25 \\
\hline Own workshop & 40.17 & 49 \\
\hline $\begin{array}{l}\text { Other work related to } \\
\text { weaving }\end{array}$ & 34.93 & 26 \\
\hline \multicolumn{3}{|l|}{ Work satisfaction } \\
\hline Yes & 60.26 & 69.61 \\
\hline No & 39.74 & 30.39 \\
\hline \multicolumn{3}{|c|}{ Any other work do for livelihood } \\
\hline Yes & 9.17 & 7 \\
\hline No & 90.83 & 93 \\
\hline \multicolumn{3}{|l|}{ Years of working } \\
\hline $15-24$ years & 44.02 & 55.88 \\
\hline $25-34$ years & 23.93 & 23.53 \\
\hline $35-45$ years & 32.05 & 20.59 \\
\hline $\begin{array}{l}\text { Mean number of years } \pm \\
\text { SD }\end{array}$ & $28.52 \pm 8.39$ & $26.40 \pm 8.17$ \\
\hline \multicolumn{3}{|l|}{ Standard of living index } \\
\hline Low & 36.75 & 26.47 \\
\hline Medium & 30.77 & 33.33 \\
\hline High & 32.48 & 40.20 \\
\hline
\end{tabular}

handloom (48.72\%) and power loom (55.88\%) workers received primary education. Majority of the handloom $(91.88 \%)$ as well as the power loom (86.27\%) workers are Muslim. Most of the handloom (74.36\%) and power loom (80.39\%) workers belong to nuclear families. The mean years of working was 28.52 years for the handloom workers and 26.40 years for the power loom workers. Majority of the handloom workers $(42.31 \%)$ had low economic status, while most of the power loom $(50.98 \%)$ workers had good economic status in work.

Table 2 shows the prevalence of MSDs in different parts of the body in the last 12 months among handloom and power loom workers. In total, $96.13 \%$ of the weavers including both handloom and power loom workers reported to have problem in any of the body region. All the handloom workers $(100 \%)$ had problem in any part and most of the problems registered by them in the last 12 months were related to upper back (84.19\%), low back (82.91\%), shoulder (76.07\%), wrist (64.53\%) and knees $(60.26 \%)$. On the other hand, $87.25 \%$ of the power loom workers had problem in any part and registered problems mostly in the low back (50\%), upper back (45.1\%), shoulder (42.16\%) and knees (35.29\%). Around $93 \%$ of the workers were disabled in the past 12 months and thus were prevented from doing normal activity.

Table 3 reports the results of logistics regression analysis examining the effects of demographic and occupational characteristics on musculoskeletal disorders in the last 12 months for various body regions. Compared to the power loom workers the handloom workers had significantly higher complaints of MSDs in neck (OR: 2.88; C.I: 1.44-5.77), shoulder (OR: 4.52; C.I.: 2.63-7.76), elbow (OR: 2.96, C.I.: 1.55-5.68), wrist (OR: 6.17, C.I.: 3.56-10.7), upper back (OR: 6.9, C.I.: 
Table 2

Prevalence and incidence of musculoskeletal disorders and disability.

\begin{tabular}{|c|c|c|c|c|}
\hline Body Regions & $\begin{array}{l}\text { Handloom } \\
\text { Worker }(\mathrm{N}= \\
234)\end{array}$ & $\begin{array}{l}\text { Powerloom } \\
\text { Worker }(\mathrm{N}= \\
102)\end{array}$ & Total & chi-2 test $\left(\chi^{2}\right)$ \\
\hline \multicolumn{5}{|l|}{ Any Problem ${ }^{a}$} \\
\hline $\begin{array}{l}\text { Past } 12 \\
\text { months }\end{array}$ & 100 & 87.25 & 96.13 & $\begin{array}{l}\text { Pearson } \chi 2(1) \\
=31.02 ; \mathrm{p}= \\
0.000\end{array}$ \\
\hline $\begin{array}{l}\text { Disabled in } \\
\text { past } 12 \\
\text { months }\end{array}$ & 99.15 & 80.39 & 93.45 & $\begin{array}{l}\text { Pearson } \chi 2(1) \\
=40.82 ; \mathrm{p}= \\
0.000\end{array}$ \\
\hline last 7 days & 57.26 & 31.37 & 49.4 & $\begin{array}{l}\text { Pearson } \chi 2(1) \\
=19.05 ; \mathrm{p}= \\
0.000\end{array}$ \\
\hline \multicolumn{5}{|l|}{ Neck } \\
\hline $\begin{array}{l}\text { Past } 12 \\
\text { months }\end{array}$ & 35.04 & 15.69 & 29.17 & $\begin{array}{l}\text { Pearson } \chi 2(1) \\
=12.88 ; \mathrm{p}= \\
0.000\end{array}$ \\
\hline $\begin{array}{l}\text { Disabled in } \\
\text { past } 12 \\
\text { months }\end{array}$ & 32.91 & 13.73 & 27.08 & $\begin{array}{l}\text { Pearson } \chi 2(1) \\
=13.23 ; \mathrm{p}= \\
0.000\end{array}$ \\
\hline last 7 days & 4.7 & 0.98 & 3.57 & $\begin{array}{l}\text { Pearson } \chi 2(1) \\
=2.85 ; \mathrm{p}= \\
0.091\end{array}$ \\
\hline \multicolumn{5}{|l|}{ Shoulder } \\
\hline $\begin{array}{l}\text { Past } 12 \\
\text { months }\end{array}$ & 76.07 & 42.16 & 65.77 & $\begin{array}{l}\text { Pearson } \chi 2(1) \\
=36.28 ; \mathrm{p}= \\
0.000\end{array}$ \\
\hline $\begin{array}{l}\text { Disabled in } \\
\text { past } 12 \\
\text { months }\end{array}$ & 67.95 & 38.24 & 58.93 & $\begin{array}{l}\text { Pearson } \chi 2(1) \\
=25.91 ; \mathrm{p}= \\
0.000\end{array}$ \\
\hline last 7 days & 20.09 & 16.67 & 19.05 & $\begin{array}{l}\text { Pearson } \chi 2(1) \\
=0.53 ; \mathrm{p}= \\
0.46\end{array}$ \\
\hline \multicolumn{5}{|l|}{ Elbows } \\
\hline $\begin{array}{l}\text { Past } 12 \\
\text { months }\end{array}$ & 31.62 & 14.71 & 26.49 & $\begin{array}{l}\text { Pearson } \chi 2(2) \\
=12.22 ; \mathrm{p}= \\
0.002\end{array}$ \\
\hline $\begin{array}{l}\text { Disabled in } \\
\text { past } 12 \\
\text { months }\end{array}$ & 20.51 & 12.75 & 18.15 & $\begin{array}{l}\text { Pearson } \chi 2(1) \\
=2.88 ; \mathrm{p}= \\
0.089\end{array}$ \\
\hline last 7 days & 3.85 & 2.94 & 3.57 & $\begin{array}{l}\text { Pearson } \chi 2(1) \\
=0.16 ; \mathrm{p}= \\
0.681\end{array}$ \\
\hline \multicolumn{5}{|l|}{ Wrists/hands } \\
\hline $\begin{array}{l}\text { Past } 12 \\
\text { months }\end{array}$ & 64.53 & 23.53 & 52.08 & $\begin{array}{l}\text { Pearson } \chi 2(1) \\
=47.84 ; \mathrm{p}= \\
0.000\end{array}$ \\
\hline $\begin{array}{l}\text { Disabled in } \\
\text { past } 12 \\
\text { months }\end{array}$ & 58.12 & 15.69 & 45.24 & $\begin{array}{l}\text { Pearson } \chi 2(1) \\
=51.63 ; \mathrm{p}= \\
0.000\end{array}$ \\
\hline last 7 days & 13.68 & 4.9 & 11.01 & $\begin{array}{l}\text { Pearson } \chi 2(1) \\
=5.57 ; \mathrm{p}= \\
0.018\end{array}$ \\
\hline \multicolumn{5}{|l|}{ Upper back } \\
\hline $\begin{array}{l}\text { Past } 12 \\
\text { months }\end{array}$ & 84.19 & 45.1 & 72.32 & $\begin{array}{l}\text { Pearson } \chi 2(1) \\
=54.22 ; \mathrm{p}= \\
0.000\end{array}$ \\
\hline $\begin{array}{l}\text { Disabled in } \\
\text { past } 12 \\
\text { months }\end{array}$ & 59.4 & 24.51 & 48.81 & $\begin{array}{l}\text { Pearson } \chi 2(1) \\
=34.61 ; \mathrm{p}= \\
0.000\end{array}$ \\
\hline last 7 days & 5.13 & 3.92 & 4.76 & $\begin{array}{l}\text { Pearson } \chi 2(1) \\
=0.22 ; \mathrm{p}= \\
0.633\end{array}$ \\
\hline \multicolumn{5}{|c|}{ Low back (small of the back) } \\
\hline $\begin{array}{l}\text { Past } 12 \\
\text { months }\end{array}$ & 82.91 & 50 & 72.92 & $\begin{array}{l}\text { Pearson } \chi 2(1) \\
=38.94 ; \mathrm{p}= \\
0.000\end{array}$ \\
\hline $\begin{array}{l}\text { Disabled in } \\
\text { past } 12 \\
\text { months }\end{array}$ & 61.11 & 38.24 & 54.17 & $\begin{array}{l}\text { Pearson } \chi 2(1) \\
=14.97 ; \mathrm{p}= \\
0.000\end{array}$ \\
\hline last 7 days & 8.55 & 3.92 & 7.14 & $\begin{array}{l}\text { Pearson } \chi 2(1) \\
=2.29 ; \mathrm{p}= \\
0.130\end{array}$ \\
\hline
\end{tabular}

Table 2 (continued)

\begin{tabular}{|c|c|c|c|c|}
\hline Body Regions & $\begin{array}{l}\text { Handloom } \\
\text { Worker }(\mathrm{N}= \\
234)\end{array}$ & $\begin{array}{l}\text { Powerloom } \\
\text { Worker }(\mathrm{N}= \\
102)\end{array}$ & Total & chi-2 test $\left(\chi^{2}\right)$ \\
\hline $\begin{array}{l}\text { Past } 12 \\
\text { months }\end{array}$ & & & & $\begin{array}{l}\text { Pearson } \chi 2(1) \\
=5.99 ; \mathrm{p}= \\
0.014\end{array}$ \\
\hline $\begin{array}{l}\text { Disabled in } \\
\text { past } 12 \\
\text { months }\end{array}$ & 9.4 & 3.92 & 7.74 & $\begin{array}{l}\text { Pearson } \chi 2(1) \\
=2.98 ; \mathrm{p}= \\
0.084\end{array}$ \\
\hline last 7 days & 5.13 & 2.94 & 4.46 & $\begin{array}{l}\text { Pearson } \chi 2(1) \\
=0.79 ; \mathrm{p}= \\
0.372\end{array}$ \\
\hline \multicolumn{5}{|c|}{ One or both knees } \\
\hline $\begin{array}{l}\text { Past } 12 \\
\text { months }\end{array}$ & 60.26 & 35.29 & 52.68 & $\begin{array}{l}\text { Pearson } \chi 2(1) \\
=17.75 ; \mathrm{p}= \\
0.000\end{array}$ \\
\hline $\begin{array}{l}\text { Disabled in } \\
\text { past } 12 \\
\text { months }\end{array}$ & 44.44 & 10.78 & 34.23 & $\begin{array}{l}\text { Pearson } \chi 2(1) \\
=35.75 ; \mathrm{p}= \\
0.000\end{array}$ \\
\hline last 7 days & 22.65 & 3.92 & 16.96 & $\begin{array}{l}\text { Pearson } \chi 2(1) \\
=17.68 ; \mathrm{p}= \\
0.000\end{array}$ \\
\hline \multicolumn{5}{|c|}{ One or both ankles/feet } \\
\hline $\begin{array}{l}\text { Past } 12 \\
\text { months }\end{array}$ & 10.68 & 4.9 & 8.93 & $\begin{array}{l}\text { Pearson } \chi 2(1) \\
=2.92 ; \mathrm{p}= \\
0.087\end{array}$ \\
\hline $\begin{array}{l}\text { Disabled in } \\
\text { past } 12 \\
\text { months }\end{array}$ & 3.42 & 0.98 & 2.68 & $\begin{array}{l}\text { Pearson } \chi 2(1) \\
=1.62 ; \mathrm{p}= \\
0.203\end{array}$ \\
\hline last 7 days & 0.85 & 0.98 & 0.89 & $\begin{array}{l}\text { Pearson } \chi 2(1) \\
=0.01 ; \mathrm{p}= \\
0.910\end{array}$ \\
\hline
\end{tabular}

${ }^{\text {a }}$ Either the neck, shoulder, elbow, wrists, upparback, low back, hips, knee, ankle.

3.79-12.54), low back (OR: 5.63; C.I.: 3.19-9.95), hip (OR: 2.18, C.I.: 1.2-3.95), knee (OR: 3.23, C.I.: 1.91-5.48) and ankle (OR: 2.97, C.I.: 1.04-8.48). The result indicates that with increasing age significantly greater problem is registered in the shoulder (OR: 1.69, C.I.: 0.99-2.88), low back (OR: 2.35, C.I.: 1.32-4.18) and knee (OR: 2.79, C.I.: 1.68-4.63). Those who owned a workshop had significantly greater problem in the elbow (OR: 2.83, C.I.: 1.39-5.79) compared to the workers. Compared to those who had work satisfaction, those without work satisfaction had significantly greater odds of having problem in the neck (OR: 2.8, C.I.: 1.49-5.12) and upper back (OR: 1.93, C.I.: 0.94-3.97).

Table 4 shows the result of logistic regression examining the effects of demographic and occupational characteristics on musculoskeletal disorders in those unable to do normal work, for various body regions in the last 12 months. It is seen from the results that the handloom workers had significantly greater odds of being unable to work due to MSD complaints in the neck (OR: 2.98; C.I.: 1.54-5.74), shoulder (OR: 3.15; C.I.: 1.91-5.28), elbow (OR: 1.88; C.I.: 0.95-3.74), wrist (OR: 7.85; C.I.: 4.27 to -14.43 ), upper back (OR: 4.91; C.I.: 2.81-8.58), low back (OR: 2.33; C.I.: 1.4-3.86), hip (OR: 2.82; C.I.: 0.91-8.77), knee (OR: 7.06; C. I.: 3.45-14.42) compared to the power loom workers. Compared to those workers in the age group of 50-54 years, those in the age-group of 56-65 years had significantly greater odds of being unable to work due to problem in the upper back (OR: 1.99; C.I.: 1.19-3.32), low back (OR:1.82; C.I.: 1.12-2.97) and knee (OR: 2.2; C.I.: 1.26-3.89). Those who did not have work satisfaction had significantly greater lost working days due to problem in the neck (OR: 2.86; C.I.: 1.52-5.63) and low back (OR: 1.63; C.I.: 0.93-2.87) compared to those who had work satisfaction. Those who did not do any other work for livelihood were significantly more likely to unable to work due to problem in neck (OR: 2.74; C.I.: 0.93-8.12) and wrist (OR: 2.75; C.I.: 1.09 - - 6.94). 
Table 3

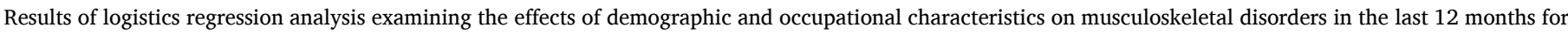
various body regions.

\begin{tabular}{|c|c|c|c|c|c|c|c|c|c|}
\hline $\begin{array}{l}\text { Background } \\
\text { Variable }\end{array}$ & Neck & Shoulder & Elbow & Wrist & Upper back & Low back & Hips & Knee & Ankle \\
\hline \multicolumn{10}{|l|}{ Weaver type } \\
\hline $\begin{array}{l}\text { Handloom } \\
\text { weavers }\end{array}$ & $\begin{array}{l}2.8^{* * *} \\
(1.47-5.22)\end{array}$ & $\begin{array}{l}4.52^{* * *} \\
(2.63-7.76)\end{array}$ & $\begin{array}{l}2.96 * * * \\
(1.55-5.68)\end{array}$ & $\begin{array}{l}6.17 * * * \\
(3.56-10.7)\end{array}$ & $\begin{array}{l}6.9^{* * *} \\
(3.79-12.54)\end{array}$ & $\begin{array}{l}5.63^{* * *} \\
(3.19-9.95)\end{array}$ & $\begin{array}{l}2.18^{* *} \\
(1.2-3.95)\end{array}$ & $\begin{array}{l}3.23 * * * \\
(1.91-5.48)\end{array}$ & $\begin{array}{l}2.97 * * \\
(1.04-8.48)\end{array}$ \\
\hline \multicolumn{10}{|c|}{$\begin{array}{l}\text { Respondent Age } \\
50 \text {-54 age }{ }^{\circledR}\end{array}$} \\
\hline $56-65$ age & $\begin{array}{l}1.5 \\
(0.82-2.56)\end{array}$ & $\begin{array}{l}1.69^{*} \\
(0.99-2.88)\end{array}$ & $\begin{array}{l}0.94 \\
(0.54-1.63)\end{array}$ & $\begin{array}{l}1.41 \\
(0.84-2.35)\end{array}$ & $\begin{array}{l}1.16 \\
(0.64-2.08)\end{array}$ & $\begin{array}{l}2.35^{* * *} \\
(1.32-4.18)\end{array}$ & $\begin{array}{l}0.53^{* *} \\
(0.31-0.9)\end{array}$ & $\begin{array}{l}2.79 * * * \\
(1.68-4.63)\end{array}$ & $\begin{array}{l}1.45 \\
(0.62-3.37)\end{array}$ \\
\hline \multicolumn{10}{|l|}{ Work Profile } \\
\hline Own workshop & $\begin{array}{l}0.5^{*} \\
(0.28-1.05)\end{array}$ & $\begin{array}{l}0.69 \\
(0.35-1.33)\end{array}$ & $\begin{array}{l}2.83^{* * *} \\
(1.39-5.79)\end{array}$ & $\begin{array}{l}1.28 \\
(0.68-2.39)\end{array}$ & $1.23(0.6-2.54)$ & $\begin{array}{l}1.22 \\
(0.6-2.45)\end{array}$ & $\begin{array}{l}0.87 \\
(0.46-1.64)\end{array}$ & $\begin{array}{l}0.98 \\
(0.53-1.81)\end{array}$ & $\begin{array}{l}0.41 * \\
(0.16-1.04)\end{array}$ \\
\hline $\begin{array}{l}\text { Other work } \\
\text { related to } \\
\text { weaving }\end{array}$ & $\begin{array}{l}0.6 \\
(0.31-1.23)\end{array}$ & $\begin{array}{l}0.75 \\
(0.36-1.56)\end{array}$ & $\begin{array}{l}1.15 \\
(0.52-2.58)\end{array}$ & $\begin{array}{l}1.15 \\
(0.59-2.24)\end{array}$ & $\begin{array}{l}1.18 \\
(0.52-2.66)\end{array}$ & $\begin{array}{l}0.8 \\
(0.38-1.67)\end{array}$ & $\begin{array}{l}0.78 \\
(0.38-1.59)\end{array}$ & $\begin{array}{l}0.95 \\
(0.49-1.83)\end{array}$ & $\begin{array}{l}0.25^{* *} \\
(0.07-0.84)\end{array}$ \\
\hline \multicolumn{10}{|c|}{$\begin{array}{l}\text { Work Satisfaction } \\
\text { Yes }{ }^{\circledR}\end{array}$} \\
\hline NO & $\begin{array}{l}2.8^{* * *} \\
(1.49-5.12)\end{array}$ & $\begin{array}{l}1.36 \\
(0.73-2.55)\end{array}$ & $\begin{array}{l}0.8 \\
(0.41-1.57)\end{array}$ & $\begin{array}{l}0.98 \\
(0.55-1.76)\end{array}$ & $\begin{array}{l}1.93^{*} \\
(0.94-3.97)\end{array}$ & $\begin{array}{l}0.82 \\
(0.43-1.55)\end{array}$ & $\begin{array}{l}0.55^{*} \\
(0.29-1.03)\end{array}$ & $\begin{array}{l}0.75 \\
(0.42-1.34)\end{array}$ & $\begin{array}{l}0.37^{*} \\
(0.12-1.11)\end{array}$ \\
\hline \multicolumn{10}{|c|}{$\begin{array}{l}\text { Any other work do for livelihood } \\
\text { Yes }(\mathbb{R})\end{array}$} \\
\hline No & $\begin{array}{l}3.1^{* *} \\
(1.03-9.06)\end{array}$ & $\begin{array}{l}1.5 \\
(0.59-3.8)\end{array}$ & $\begin{array}{l}0.31 * * \\
(0.13-0.72)\end{array}$ & $\begin{array}{l}1.96 \\
(0.82-4.7)\end{array}$ & $\begin{array}{l}0.27 * \\
(0.07-1.08)\end{array}$ & $\begin{array}{l}1.67 \\
(0.66-4.25)\end{array}$ & $\begin{array}{l}0.71 \\
(0.28-1.77)\end{array}$ & $\begin{array}{l}3.23^{* *} \\
(1.32-7.9)\end{array}$ & $\begin{array}{l}0.2^{* * *} \\
(0.06-0.6)\end{array}$ \\
\hline \multicolumn{10}{|c|}{$\begin{array}{l}\text { Years of working } \\
11-24 \text { years } \mathbb{R}\end{array}$} \\
\hline 25-34 years & $\begin{array}{l}0.9 \\
(0.44-1.78)\end{array}$ & $\begin{array}{l}2.72^{* * *} \\
(1.44-5.13)\end{array}$ & $\begin{array}{l}0.98 \\
(0.51-1.89)\end{array}$ & $\begin{array}{l}0.93 \\
(0.51-1.69)\end{array}$ & $\begin{array}{l}2.66^{* *} \\
(1.33-5.35)\end{array}$ & $\begin{array}{l}1.86^{*} \\
(0.96-3.6)\end{array}$ & $\begin{array}{l}0.74 \\
(0.39-1.37)\end{array}$ & $\begin{array}{l}0.64 \\
(0.35-1.15)\end{array}$ & $\begin{array}{l}1.57 \\
(0.59-4.18)\end{array}$ \\
\hline 35-45 years & $\begin{array}{l}1.4 \\
(0.79-2.63)\end{array}$ & $\begin{array}{l}4.36 * * * \\
(2.18-8.72)\end{array}$ & $\begin{array}{l}1.4 \\
(0.75-2.62)\end{array}$ & $\begin{array}{l}1.7^{*} \\
(0.94-3.07)\end{array}$ & $\begin{array}{l}10.47^{* * *} \\
(4.06-27.04)\end{array}$ & $\begin{array}{l}3.35^{* * *} \\
(1.59-7.06)\end{array}$ & $\begin{array}{l}0.61 \\
(0.32-1.16)\end{array}$ & $\begin{array}{l}1.15 \\
(0.65-2.04)\end{array}$ & $\begin{array}{l}1.22 \\
(0.47-3.19)\end{array}$ \\
\hline
\end{tabular}

Note: ${ }^{*} \mathrm{p}<0.1,{ }^{*} \mathrm{p}<0.05, * * * \mathrm{p}<0.01$. ${ }^{\circledR}$ reference category.

Table 4

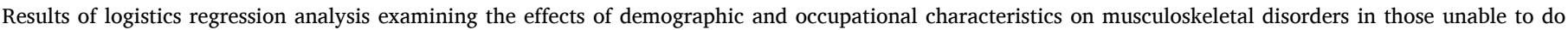
normal work, for various body regions in the last 12 months.

\begin{tabular}{|c|c|c|c|c|c|c|c|c|c|}
\hline $\begin{array}{l}\text { Background } \\
\text { Variable }\end{array}$ & Neck & Shoulder & Elbow & Wrist & Upper back & Low back & Hips & Knee & Ankle \\
\hline \multicolumn{10}{|c|}{ Powerloom weavers ${ }^{\circledR}$} \\
\hline $\begin{array}{l}\text { Handloom } \\
\text { weavers }\end{array}$ & $\begin{array}{l}2.98 * * * \\
(1.54-5.74)\end{array}$ & $\begin{array}{l}3.18^{* * *} \\
(1.91-5.28)\end{array}$ & $\begin{array}{l}1.88^{*} \\
(0.95-3.74)\end{array}$ & $\begin{array}{l}7.85^{* * *} \\
(4.27-14.43)\end{array}$ & $\begin{array}{l}4.91 * * * \\
(2.81-8.58)\end{array}$ & $\begin{array}{l}2.33^{* * *} \\
(1.4-3.86)\end{array}$ & $\begin{array}{l}2.82^{*} \\
(0.91-8.77)\end{array}$ & $\begin{array}{l}7.06^{* * *} \\
(3.45-14.42)\end{array}$ & $\begin{array}{l}4.54 \\
(0.51-40.12)\end{array}$ \\
\hline \multicolumn{10}{|c|}{ Respondent Age } \\
\hline $56-65$ age & $\begin{array}{l}1.4 \\
(0.79-2.5)\end{array}$ & $\begin{array}{l}1.07 \\
(0.65-1.76)\end{array}$ & $\begin{array}{l}0.97 \\
(0.53-1.79)\end{array}$ & $\begin{array}{l}1.21 \\
(0.72-2.04)\end{array}$ & $\begin{array}{l}1.99 * * \\
(1.19-3.32)\end{array}$ & $\begin{array}{l}1.82^{* *} \\
(1.12-2.97)\end{array}$ & $\begin{array}{l}1.15 \\
(0.46-2.84)\end{array}$ & $\begin{array}{l}2.21 * * \\
(1.26-3.89)\end{array}$ & $\begin{array}{l}2.07 \\
(0.43-9.93)\end{array}$ \\
\hline \multicolumn{10}{|l|}{ Work Profile } \\
\hline Own workshop & $\begin{array}{l}0.69 \\
(0.35-1.37)\end{array}$ & $\begin{array}{l}0.67 \\
(0.37-1.25)\end{array}$ & $\begin{array}{l}2.99 * * \\
(1.31-6.83)\end{array}$ & $\begin{array}{l}0.94 \\
(0.5-1.78)\end{array}$ & $\begin{array}{l}1.93 * * \\
(1.03-3.61)\end{array}$ & $\begin{array}{l}0.66 \\
(0.37-1.2)\end{array}$ & $\begin{array}{l}0.88 \\
(0.29-2.64)\end{array}$ & $\begin{array}{l}0.68 \\
(0.34-1.36)\end{array}$ & $\begin{array}{l}0.37 \\
(0.06-2.22)\end{array}$ \\
\hline $\begin{array}{l}\text { Other work } \\
\text { related to } \\
\text { weaving }\end{array}$ & $\begin{array}{l}0.7 \\
(0.35-1.41)\end{array}$ & $\begin{array}{l}0.92 \\
(0.47-1.8)\end{array}$ & $\begin{array}{l}1.25 \\
(0.5-3.17)\end{array}$ & $\begin{array}{l}0.92 \\
(0.47-1.8)\end{array}$ & $\begin{array}{l}1.77^{*} \\
(0.91-3.45)\end{array}$ & $\begin{array}{l}0.96 \\
(0.5-1.84)\end{array}$ & $\begin{array}{l}1.07 \\
(0.32-3.59)\end{array}$ & $\begin{array}{l}1.66 \\
(0.81-3.42)\end{array}$ & $\begin{array}{l}0.57 \\
(0.09-3.61)\end{array}$ \\
\hline \multicolumn{10}{|c|}{ Work Satisfaction } \\
\hline No & $\begin{array}{l}2.86 * * * \\
(1.52-5.36)\end{array}$ & $\begin{array}{l}1.27 \\
(0.71-2.27)\end{array}$ & $\begin{array}{l}1.07 \\
(0.5-2.26)\end{array}$ & $\begin{array}{l}0.87 \\
(0.48-1.57)\end{array}$ & $\begin{array}{l}1.23 \\
(0.69-2.21)\end{array}$ & $\begin{array}{l}1.63^{*} \\
(0.93-2.87)\end{array}$ & $\begin{array}{l}0.55 \\
(0.18-1.66)\end{array}$ & $\begin{array}{l}0.36^{* * *} \\
(0.18-0.71)\end{array}$ & $\begin{array}{l}0.35 \\
(0.05-2.36)\end{array}$ \\
\hline \multicolumn{10}{|c|}{$\begin{array}{l}\text { Any other work do for livelihood } \\
\text { Yes } \mathbb{R}\end{array}$} \\
\hline No & $\begin{array}{l}2.74^{*} \\
(0.93-8.12)\end{array}$ & $\begin{array}{l}1.87 \\
(0.8-4.41)\end{array}$ & $\begin{array}{l}0.59 \\
(0.23-1.53)\end{array}$ & $\begin{array}{l}2.75^{* *} \\
(1.09-6.94)\end{array}$ & $\begin{array}{l}0.52 \\
(0.21-1.31)\end{array}$ & $\begin{array}{l}1.75 \\
(0.74-4.14)\end{array}$ & $\begin{array}{l}0.27^{* *} \\
(0.08-0.89)\end{array}$ & $\begin{array}{l}2.01 \\
(0.75-5.35)\end{array}$ & $\begin{array}{l}0.14^{* *} \\
(0.03-0.74)\end{array}$ \\
\hline \multicolumn{10}{|c|}{ Years of working } \\
\hline $25-34$ years & $\begin{array}{l}0.89 \\
(0.44-1.81)\end{array}$ & $\begin{array}{l}1.79 * \\
(0.99-3.23)\end{array}$ & $\begin{array}{l}0.82 \\
(0.39-1.71)\end{array}$ & $\begin{array}{l}0.94 \\
(0.51-1.74)\end{array}$ & $\begin{array}{l}1.47 \\
(0.81-2.68)\end{array}$ & $\begin{array}{l}1.59 \\
(0.89-2.83)\end{array}$ & $\begin{array}{l}0.19 * * \\
(0.04-0.85)\end{array}$ & $\begin{array}{l}0.92 \\
(0.47-1.82)\end{array}$ & $\begin{array}{l}1.67 \\
(0.32-8.71)\end{array}$ \\
\hline $35-45$ years & $\begin{array}{l}1.47 \\
(0.8-2.69)\end{array}$ & $\begin{array}{l}2.51 * * * \\
(1.39-4.53)\end{array}$ & $\begin{array}{l}1.22 \\
(0.61-2.42)\end{array}$ & $\begin{array}{l}1.38 \\
(0.77-2.49)\end{array}$ & $\begin{array}{l}1.76^{*} \\
(0.98-3.15)\end{array}$ & $\begin{array}{l}1.66^{*} \\
(0.94-2.92)\end{array}$ & $\begin{array}{l}0.39 * \\
(0.13-1.13)\end{array}$ & $\begin{array}{l}1.93 * * \\
(1.06-3.53)\end{array}$ & $\begin{array}{l}0.69 \\
(0.12-4.09)\end{array}$ \\
\hline
\end{tabular}

Note: ${ }^{*} \mathrm{p}<0.1,{ }^{* *} \mathrm{p}<0.05,{ }^{* *} \mathrm{p}<0.01$. ${ }^{\circledR}$ reference category. 


\section{Discussion}

Musculoskeletal disorder is a graving issue among the informal sectors, and the handloom industry is no exception to this. Working for long hours, sitting in a static posture make the workers vulnerable to MSDs. In view of this, the present study aims to investigate the prevalence of MSDs among the weaving community, which includes workers at both handlooms and power looms. Bivariate analysis suggested a high prevalence of MSD among the handloom and power loom workers. The problems encompassed by the handloom workers is more in the upper and lower back, shoulder and the wrist/hands. MSDs preventing regular activities were significantly higher among handloom workers compared to power loom workers. This may be because hand loom workers have to sit for long hours and work with their hands engaging their wrist compared to the power looms where most of the work done is machine operated. $^{3}$

The health issues reported by the handloom workers are similar to issues reported by various other sectors, including the metal industry, construction work, mining industry, and spinning industry. ${ }^{19,22}$ Literature particular to the carpet weaving industry have also indicated a high prevalence of musculoskeletal symptoms due to various working factors like postures, loom type, working hours, poor lighting and ambient conditions, poor air quality, lack of work-rest regime and seat type, which led to loss of more than fifteen-thousand-man days and less productivity in subsequent years of employment. ${ }^{9,10,23}$ Earlier studies on handloom workers of Bangladesh and Nepal also showed similar problems, and the major affected areas were neck, shoulder, back and wrist. ${ }^{16,24}$ Studies in the Indian context on workers of handloom and power looms in the textile industry of India reported that both male and female workers had significant MSD related to work. ${ }^{2,12}$ The most prominent MSDs among weavers were shoulder's pain, backache, pain in palm $\&$ joints and spondylitis. ${ }^{12}$ In the study of North East Indian handloom workers, muscular diseases, fatigue, impeded blood circulation and venous blood pooling, in-vertebral joint laxity and fluid loss in the spine hamstring muscles were found. ${ }^{13}$

Disease pattern is also related to age and occupation duration. Our study found that with an increase in the years of working, the prevalence of MSDs increases. This finding is similar to earlier study based on handloom weavers of Kerala, which indicates that MSD prevalence is different at different age groups and increases with the duration of work. Workers working in the dying sector have the highest risk of MSDs, followed by the weaving, winding, and finishing sector workers. The study also concluded that light, heat, noise, dust, and odour also affect the MSDs among the workers in the industry. ${ }^{20}$ Handloom weavers in West Bengal also suffered from lower back pain, and this is directly related to the number of years of working experience. ${ }^{25}$

The handloom workers of Varanasi district are prone to MSDs related to the wrist, shoulder, upper and lower back. These conditions deteriorate with increased age as well as increasing years of employment. They work in the makeshift arrangements within their houses, and the tradition is passed on from one generation to the other. They are not protected by any occupational health and safety measures and are not entitled to any benefits or job security. Their lower socio-economic position and poor housing conditions also increase their health risks.

\section{Strategies to minimise the burden of MSDs among the weaving community}

The study recommends some preventive measures to minimise the prevalence of MSDs among the study population. The weavers have to be made aware that MSD is not life taking disorder and could be minimized through exercise and stretching activities. The health providers can play an active role to minimise the burden through educating these people about the early signs of MSDs, and promoting the need of physical exercises to minimise the pains related to MSDs. Since most of the study population are from low socio-economic background and do not have enough to meet the health care needs, they should be made aware of all the central and state sponsored health care schemes and should effectively utilise the schemes. Moreover, there is a need to improve the work environment with proper lighting and sitting arrangement. The work hours should also be restricted to few hours and they should not work continuously sitting in static position.

\section{Limitations of the study}

The present study has used a cross-sectional survey design to gather data. Using a cross-sectional survey to collect data could have resulted in underestimating the true prevalence of MSDs. Since the MSD prevalence was based on self-reporting, this could have led to recall bias. Moreover, MSD severity was not quantified. Data were collected from handloom and power loom weavers of Varanasi district of India, and generalisation of the results to similar occupations must be made carefully. Moreover, the respondents were mostly aged 50 years and are more susceptible to MSDs than the young workers, and thus generalisation of the results to all the handloom and power loom weavers must be made with caution.

\section{Scope for future research}

The present study has highlighted the prevalence of MSD among the weavers. However, it has not explored the inpatient and outpatient health care costs. Thus future study should focus on the cost of meeting the health care needs, the coping mechanism and the economic burden of MSDs among the weavers.

\section{Availability of data and materials}

The data used for the study is primarily collected for the PhD thesis work during November 2019 to February 2020 in Varanasi (UP), India. The ethics statement and consent for publication is applicable of the Ethical approval of the institute research committee.

\section{Funding}

No funding for this study.

\section{Informed consent}

Respondent oral and written consent was applicable.

\section{Authors contribution}

Laeek Ahemad Siddiqui: Conceptualization, develop questionnaire, collected data, Data analysis. Adrita Banerjee: writing of the manuscript. Praveen Kailash Chokhandre: Review and edited manuscript Sayeed Unisa: in review the discussion and finalization of manuscript.

\section{Declaration of competing interest}

The authors have declared that no competing interest exists.

\section{Acknowledgements}

Authors acknowledge the time and support given by study participants.

\section{References}

1 Koiri P. Occupational health problems of the handloom workers : a cross sectional study of Sualkuchi, Assam, Northeast India. Clin Epidemiol Glob Heal. 2020;8(4): 1264-1271. https://doi.org/10.1016/j.cegh.2020.04.025.

2 Nag A, Vyas H, Nag P. Occupational health scenario of Indian informal sector. 2016: 377-385. Published online. 
3 Awasthi S, Singh P, Awasthi N. In: Risk assessment of handloom weavers for musculoskeletal disorder in durrie unit. 7. 2018:94-98, 7.

4 Eurofound. 6th European Working Conditions Survey : 2017 Update. 2017.

5 Guo HR, Chang YC, Yeh WY, Chen CW, Guo YL. Prevalence of musculoskeletal disorder among workers in taiwan: a nationwide study. $J$ Occup Health. 2004;46(1): 26-36. https://doi.org/10.1539/joh.46.26.

6 Gerr F, Marcus M, Ensor C, et al. A prospective study of computer users: I. Study design and incidence of musculoskeletal symptoms and disorders. Am J Ind Med. 2002;41(4):221-235. https://doi.org/10.1002/ajim.10066.

7 WHO. The burden of musculoskeletal conditions at the start of the new millennium. World Health Organ Tech Rep Ser. 2003:919.

8 Hossain A, Kamrujjaman M, Maleque A. Associated factors \& pattern of musculoskeletal pain among male handloom weavers residing in belkuchi, shirajganj: a cross sectional study. Int J Sci Eng Res. 2018;9(10):1447-1451.

9 Choobineh A, Lahmi M, Shahnavaz H, Khani R, Hosseini M. Musculoskeletal Symptoms as Related to Ergonomic Factors in Iranian Hand-Woven Carpet Industry and General Guidelines for Workstation Design Musculoskeletal Symptoms as Related to Ergonomic Factors in Iranian Hand-Woven Carpet Industry and General Guideli. vol. 2007. February 2016:3548. https://doi.org/10.1080/10803548.2004.11076604.

10 Choobineh A, Hosseini M, Lahmi M. Weaving Posture Analyzing System (WEPAS): Introduction and Validation. vol. 34. 2004:139-147. https://doi.org/10.1016/j. ergon.2004.03.004.

11 Ramdan IM, Candra KP, Fitri AR. Factors affecting musculoskeletal disorder (MSD) prevalence among women weavers working with handlooms in samarinda, Indonesia. Int J Occup Saf Ergon. 2018:1-23. https://doi.org/10.1080/ 10803548.2018.1481564, 0(0).

12 Goel A, Tyagi I. OCCUPATIONAL HEALTH HAZARDS RELATED TO WEAVING. vol. 1. 2012:22-28, 1 .

13 Pandit S, Kumar P, Chakrabarti D. In: Ergonomic Problems Prevalent in Handloom Units of. 3. 2013:1-7, 1.

14 Textiles M. Of. Fourth all India handloom census 2019-20. Published online Minist Text Gov India. 2019:277, 01-.
15 Kuorinka I, Jonsson B, Kilbom A, et al. Standardised Nordic questionnaires for the analysis of musculoskeletal symptoms. Appl Ergon. 1987;18(3):233-237. https://doi. org/10.1016/0003-6870(87)90010-x.

16 Rahman M, Khan MH, Hossain I, Bari S. Musculoskeletal problems among handloom workers. Texila Int J. September 2017;2017(5), 0-15.

17 Singh S, Chokhandre P. Assessing the impact of waste picking on musculoskeletal disorders among waste pickers in Mumbai, India : a cross-sectional study. 2015:1-8. https://doi.org/10.1136/bmjopen-2015-008474. Published online.

18 Salve PS, Chokhandre P. Assessing the exposure of street sweeping and potential risk factors for developing musculoskeletal disorders and related disabilities : a cross-sectional study. 2016:1-8. https://doi.org/10.1136/bmjopen-2016-012354. Published online.

19 Lagerstrom M. Wenemark M. p Occupational and individual factors related to musculoskeletal symptoms in five body regions among Swedish nursing personnel. 1995: 27-35. Published online.

20 Satheeshkumar M, Krishnakumar K. WORK-RELATED musculoskeletal disorders among handloom workers IN Kerala, India. Int J Adv Res Eng Technol. 2020;11(6): 826-835. https://doi.org/10.34218/IJARET.11.6.2020.074.

21 Neeraja T, Bhargavi A, Manjulatha C. Musculoskeletal disorders and visual strain among handloom weavers. Int J Inf Retr Res (IJIRR). 2016;(10):2942-2945, 03 http://www.ijirr.com/sites/default/files/issues-files/1359_0.pdf.

22 Gamperiene M, Stigum H. Work related risk factors for musculoskeletal complaints in the spinning industry in Lithuania. 1999:411-416. Published online.

23 Motamedzade M, Moghimbeigi A. Musculoskeletal Disorders Among Female Carpet Weavers in Iran. 2012. https://doi.org/10.1080/00140139.2011.582539, 0139.

24 Paudyal P, Ayres JG, Semple S, Macfarlane GJ. Low Back Pain Among Textile Workers : A Cross-Sectional Study. 2013:129-134. https://doi.org/10.1093/occmed/kqs231. January.

25 Durlov S, Chakrabarty S, Chatterjee A, et al. Prevalence of low back pain among handloom weavers in West Bengal, India. 2014:333-339. https://doi.org/10.1179/ 2049396714Y.0000000082. Published online. 